\title{
Can adult ASD outcomes be predicted by clinical measures made during childhood?
}

\author{
By Jessica K. Edwards
}

A new study has retrospectively profiled the adult outcomes of 123 children with autism spectrum disorder (ASD) to determine whether certain outcomes, or "types", can be predicted from typical clinical measures made during childhood. The researchers used a set of 15 variables to characterise adult outcomes, including the verbal and non-verbal IQ level, ASD symptom severity score, behavioural problems and medication use. From these, they found that only four classes of adult outcomes existed in their population: one good, one intermediate and two low-functioning classes (in which one had co-occurring behavioural problems). These classes could be confidently predicted from childhood verbal IQ levels and autism symptom severity recorded from age two to nine years. The accuracy of the prediction improved over time. Interestingly, removing verbal and non-verbal IQ and autism symptom severity measurements from the set of 15 variables had no effect on the number of the latent classes; however, the class composition changed and the class prediction accuracy diminished.
The researchers suggest that these data might help clinicians develop goals with individuals with ASD and do forward planning with respect to transition into adult mental health services. They do, however, urge caution in using these four classes as an aetiological taxonomy. This typology gave weight to cognitive outcomes while outcomes concerning subjective well-being and the affective domain were poorly predicted from childhood measures. Future research is thus needed to understand how to balance subjective measures of well-being and objective measures of functioning to describe positive adaptations in adulthood.

\section{Referring to:}

Pickles, A., McCauley, J.B., Pepa, L.A., Huerta, M. \& Lord, C. (2020), The adult outcome of children referred for autism: typology and prediction from childhood. J. Child Psychol. Psychiatry. doi: 10.111/ jcpp.13180. 